\title{
Glucose transporter GLUT1 expression is an stage-independent predictor of clinical outcome in adrenocortical carcinoma
}

\begin{abstract}
Wiebke Fenske ${ }^{1 *}$, Hans-Ullrich Völker ${ }^{2 *}$, Patrick Adam ${ }^{2}$, Stefanie Hahner ${ }^{1}$, Sarah Johanssen ${ }^{1}$, Sebastian Wortmann ${ }^{1}$, Melanie Schmidt ${ }^{3}$, Michael Morcos ${ }^{4}$, Hans-Konrad Müller-Hermelink ${ }^{2}$, Bruno Allolio ${ }^{1}$ and Martin Fassnacht ${ }^{1}$
\end{abstract}

\footnotetext{
${ }^{1}$ Endocrine and Diabetic Unit, Department of Internal Medicine I, University Hospital of Würzburg and ${ }^{2}$ Institute of Pathology, University of Würzburg, Josef-Schneider-Strasse 2, D-97080 Würzburg, Germany

${ }^{3}$ Department of Gynaecology, University Hospital of Würzburg, Würzburg, Germany

${ }^{4}$ Department of Internal Medicine I, University Hospital, University of Heidelberg, Heidelberg, Germany

(Correspondence should be addressed to M Fassnacht; Email: fassnacht_m@medizin.uni-wuerzburg.de)

*(W Fenske and H-U Völker contributed equally to this work)
}

\begin{abstract}
Owing to the rarity of adrenocortical carcinoma (ACC) no prognostic markers have been established beyond stage and resection status. Accelerated glycolysis is a characteristic feature of cancer cells and in a variety of tumour entities key factors in glucose metabolism like glucose transporter 1 and 3 (GLUT1 and -3), transketolase like-1 enzyme (TKTL1) and pyruvate kinase type M2 (M2-PK) are overexpressed and of prognostic value. Therefore, we investigated the role of these factors in ACC. Immunohistochemical analysis was performed on tissue microarrays of paraffin-embedded tissue samples from 167 ACCs, 15 adrenal adenomas and 4 normal adrenal glands. Expression was correlated with baseline parameters and clinical outcome. GLUT1 and -3 were expressed in 33 and $17 \%$ of ACC samples respectively, but in none of the benign tumours or normal adrenals glands. By contrast, TKTL1 and M2-PK were detectable in all benign tissues and the vast majority of ACCs. GLUT1 expression was strongly associated with prognosis in univariate and multivariate analysis $(P<0.01)$, whereas GLUT3, TKTL1 and M2-PK did not correlate with clinical outcome. Patients with strong GLUT1 staining showed a considerably higher overall mortality (hazard ratio (HR) 6.34 (95\% confidence interval 3.10-12.90) compared with patients with no GLUT1 staining. When analysing patients in their early stages and advanced disease separately, similar results were obtained. HR for survival was 5.31 (1.80-15.62) in patients with metatastic ACC and in patients after radical resection the HR for disease-free survival was 6.10 (2.16-16.94). In conclusion, GLUT1 is a highly promising stage-independent, prognostic marker in ACC.
\end{abstract}

Endocrine-Related Cancer (2009) 16 919-928

\section{Introduction}

Adrenocortical carcinoma (ACC) is a rare disease characterised by an aggressive behaviour with an overall 5-year survival ranging from 16 to $44 \%$ (Dackiw et al. 2001, Allolio et al. 2004, Allolio \& Fassnacht 2006, Libe et al. 2007, Fassnacht et al. 2009). The precise pathogenesis of ACC is still poorly understood and due to the rarity of the disease prognostic assessment and treatment strategies are not well standardised (Schteingart et al. 2005).
Many patients present with locally advanced or metastatic disease and up to $85 \%$ of patients with radically resected tumours relapse (Allolio \& Fassnacht 2006, Fassnacht et al. 2006). Disease stage and completeness of initial resection are currently the only widely accepted determinants of outcome for this disease (Barzon et al. 1997, Vassilopoulou-Sellin \& Schultz 2001, Schteingart et al. 2005, Fassnacht et al. 2009). However, these features are poorly applicable when fronting patients at a given stage and only partly reflect 
the wide prognostic heterogeneity of the disease. Therefore, histological and molecular features for prediction of tumour behaviour would be of major importance for tailored therapeutic decisions. However, till date reliable prognostic factors for survival in ACC are lacking.

More than 80 years ago, Warburg (1956) described that one of the most fundamental characteristics of cancer cells is an increased level of glucose uptake and its anaerobic metabolism. Most recently, several key factors in glucose metabolism have been described to be overexpressed in different human carcinomas (for e.g. glioblastoma, colorectal, gastric, pancreatic and breast cancer (Reske et al. 1997, Grover-McKay et al. 1998, Urano et al. 2006, Wincewicz et al. 2007). Especially, the hypoxiaresponsive glucose transporters isoforms 1 and 3 (GLUT1 and -3) have been shown to be associated with shortened survival in several tumour entities (as in colon, urethelial, oral squamous cell, and head and neck carcinomas (Smith 1999, Langbein et al. 2006, Eckert et al. 2008, Zhou et al. 2008)) and may possibly assist in the selection of patients for more aggressive therapy. In addition, high expression of transketolase like-1 enzyme (TKTL1) in tumour cells is correlated with poor clinical outcome in colon and urothelial cancer (Langbein et al. 2006). TKTL1 encodes an alternative transketolase transcript, opening a new energy source for the tumour cell by enabling oxygen-independent glucose degradation and allowing glucose conversion to ribose for nucleic acid synthesis (Coy et al. 2005). Specific transketolase inhibitors slow down tumour cell proliferation (Comin-Anduix et al. 2001), whereas the activation of transketolase stimulates tumour growth (Rais et al. 1999). In many tumour cells, the pyruvate kinase equilibrium is dysregulated at the cost of ATP production by expression of the dimeric pyruvate kinase type M2 (M2-PK). This mechanism allows tumour cells to invade areas with low oxygen and glucose concentrations (Mazurek et al. 2005) and might be relevant for the clinical behaviour of the tumour (Yoo et al. 2004).

Owing to the rarity of ACC, single centres were not able to collect an adequate sample of tumours for investigating the role of glucose metabolism in ACC carcinogenesis and to correlate factors in glucose metabolism with clinical outcome. Owing to the efforts of the German ACC Registry Group, we were able to collect 167 clinically annotated ACC tumour samples. In the present study, we have used tissue microarrays (TMAs) and immunohistochemistry analysis to investigate expression of GLUT1, -3,
TKTL1 and M2-PK in patients with ACC, with benign adrenal adenomas, and in normal adrenal glands. In addition, we have analysed the prognostic value of these markers in ACC.

\section{Material and methods}

\section{Clinical data and specimen}

A total of 186 adrenocortical tissues were collected from patients undergoing surgery for ACC $(n=167)$, aldosterone-producing adenoma $(n=5)$, cortisolproducing adenoma $(n=5)$ and endocrine inactive adenomas $(n=5)$. The normal adrenal tissue was derived from adrenal glands removed as part of tumour nephrectomy $(n=4)$. Table 1 summarises the characteristics of patients and tissue samples included in this study. A total of 134 ACC samples were derived from surgery of the primary tumour; 19 of local recurrence and 14 of distant metastasis. The diagnosis of ACC was based on established clinical, biochemical and morphological criteria (Allolio \& Fassnacht 2006). All histological diagnoses were confirmed by the reference pathologist of the German ACC Registry (Wolfgang Saeger, Hamburg, Germany) and malignancy was established by a Weiss score $\geq 3$ (Weiss et al. 1989). Clinical data of ACC patients, including follow-up and survival data, were collected in a structured manner by the German ACC Registry (www.nebennierenkarzinom.de; Koschker et al. 2006, Fassnacht et al. 2009). Tumour staging was based on imaging studies and findings during surgery, and was reported according to the UICC/WHO classification 2004 (DeLellis et al. 2004). Owing to the retrospective data collection, pre-surgical endocrine work-up and imaging intervals during follow-up were not standardised. However, in the majority of patients glucocorticoid and/or androgen excess was investigated with at least three of the following tests: serum cortisol; plasma ACTH; 24-h urinary free cortisol; $1 \mathrm{mg}$ dexamethasone suppression test; serum dehydroepiandrosteronsulfat (DHEA-S); androstendione; 17-OH progesterone; and testosterone (Fassnacht et al. 2004). Surgery of the primary tumour was judged as radical resection if surgical, pathological and imaging; reports did not provide evidence for macroscopically remaining disease. The database for follow-up information was locked in May 2008.

Patients gave informed consent for collecting tissue and clinical data and the study was approved by the ethics committee of the University of Würzburg (Germany, board approval number 92/02 and 86/03). 


\begin{tabular}{|c|c|c|c|}
\hline & Age (years) & $\operatorname{Sex}(M / F)$ & $\begin{array}{l}\text { Primary tumour } \\
\text { size }(\mathrm{cm})\end{array}$ \\
\hline \multicolumn{4}{|l|}{ ACC } \\
\hline Primary tumour $(n=134)$ & $49(16)$ & $(48 / 86)$ & $12(4.4)$ \\
\hline $\mathrm{WHO}$ I $(n=5)^{\mathrm{a}}$ & $54(24)$ & $(2 / 3)$ & $4.5(0.2)$ \\
\hline WHO II $(n=53)$ & $48(16)$ & $(20 / 33)$ & $11.5(4.4)$ \\
\hline WHO III $(n=26)$ & $52(14)$ & $(10 / 16)$ & $11.4(3.8)$ \\
\hline WHO IV $(n=45)$ & $49(17)$ & $(14 / 31)$ & $13.4(4)$ \\
\hline Local recurrence $(n=19)^{\mathrm{b}}$ & $46(17)$ & $(9 / 10)$ & - \\
\hline Distant metastases $(n=14)^{\mathrm{b}}$ & $44(11)$ & $(3 / 11)$ & - \\
\hline \multicolumn{4}{|l|}{ Adenoma } \\
\hline Aldosterone-producing adenoma $(n=5)$ & $47(14)$ & $2 / 3$ & $2.0(0.1)$ \\
\hline Cortisol-producing adenoma $(n=5)$ & $52(22)$ & $0 / 5$ & $2.6(0.3)$ \\
\hline Inactive adenoma $(n=5)$ & $67(20)$ & $2 / 3$ & $1.8(0.3)$ \\
\hline Normal adrenal gland $(n=4)$ & $63(18)$ & $3 / 1$ & - \\
\hline
\end{tabular}

Data are mean (S.D.) or numbers. WHO, World Health Organization/international union against cancer UICC; $f$, female; $m$, male. an six cases tumour stage was not determined.

${ }^{b}$ Nineteen out of the 33 samples from surgery for recurrence or distant metastases derived from patients with more than one analysed sample.

\section{Tissue microarray}

A total of 182 different adrenocortical tumours (167 carcinomas and 15 benign lesions) and 4 normal adrenal samples were assembled into three TMAs. Briefly, haematoxylin and eosin stained sections of formalin-fixed and paraffin-embedded tissue blocks were re-evaluated to identify the representative areas of well-preserved morphology (without significant necrotic tissue). The corresponding area on the block was marked for tissue punching. The TMA was assembled using a commercially available manual tissue puncher/ arrayer (Beecher Instruments, Silver Spring, MD, USA) according to the manufacturer's instructions. Five cores with a diameter of $0.6 \mathrm{~mm}$ were punched from each tissue block and arrayed into a recipient paraffin block at a distance of $1 \mathrm{~mm}$ between each core. We chose to array five punch biopsies per case to minimise the number of cases not analysable due to tissue loss and to increase concordance rates among different cores. Sections $(2 \mu \mathrm{m})$ were cut on silanised slides (Superfrost, Langenbrinck, Emmendingen, Germany) also used for conventional immunohistochemical stains. Before staining, slides were dried at room temperature for 7 days in order to minimise tissue loss. For each block, haematoxylin and eosin stained slides were cut to verify the tumour cell content.

\section{Immunohistochemical staining}

Immunohistochemical staining with commercial available antibodies was done in standard technique following the manufacturer's protocols. Positive controls were used in accordance with the manufacturer's recommendations or published experience (Table 2). TMA slides were deparaffinised with xylene. Sections of positive controls were stained simultaneously. Rinsing the slides in decreasing concentrations of ethanol was followed by antigen unmasking in $10 \mathrm{mM}$ sodium citrate buffer $(\mathrm{pH}=6.0)$ in a microwave oven at

Table 2 Used antibodies, source and dilution

\begin{tabular}{|c|c|c|c|c|c|}
\hline Antibody & Stained protein & Clone/species & Source & Dilution & Positive control \\
\hline GLUT1 & $\begin{array}{l}\text { Glucose transporter } 1, \\
\text { localised membraneously } \\
\text { and cytoplasmatically }\end{array}$ & Polyclonal rabbit & $\begin{array}{l}\text { DAKO, Hamburg, } \\
\text { Germany }\end{array}$ & $1: 100$ & Epidermis \\
\hline GLUT3 & $\begin{array}{l}\text { Glucose transporter } 1 \\
\text { localised membraneously }\end{array}$ & Polyclonal rabbit & $\begin{array}{l}\text { LAB VISION, Warm } \\
\text { Springs, Fremont, } \\
\text { CA, USA }\end{array}$ & $1: 500$ & $\begin{array}{l}\text { Laryngeal squamous } \\
\text { cell carcinoma }\end{array}$ \\
\hline M2-PK & $\begin{array}{l}\text { M2 form of pyruvate kinase, } \\
\text { localised cytoplasmatically }\end{array}$ & DF4 & $\begin{array}{l}\text { ScheBo Biotech, } \\
\text { Gießen, Germany }\end{array}$ & $1: 250$ & $\begin{array}{l}\text { Colorectal } \\
\text { adenocarcinoma }\end{array}$ \\
\hline TKTL1 & $\begin{array}{l}\text { Transketolase isoform TKTL-1, } \\
\text { localised cytoplasmatically }\end{array}$ & $\begin{array}{l}\text { JFC12T10 } \\
\text { monoclonal } \\
\text { mouse }\end{array}$ & $\begin{array}{l}\text { Linaris, Wertheim, } \\
\text { Germany }\end{array}$ & $1: 200$ & $\begin{array}{l}\text { Colorectal } \\
\text { adenocarcinoma }\end{array}$ \\
\hline
\end{tabular}


$600 \mathrm{~W}$ for 5 min. After rinsing in distilled $\mathrm{H}_{2} \mathrm{O}$, endogenous peroxidase was inhibited by incubation for $10 \mathrm{~min}$ in $3 \% \mathrm{H}_{2} \mathrm{O}_{2}$ in methanol. Slides were washed with PBS and incubated with $1 \%$ goat serum in PBS for $15 \mathrm{~min}$. Subsequently, slides were incubated with the different antibodies diluted in antibody diluents (DAKO, Hamburg, Germany; Table 2).

After 45-60 min. of incubation at room temperature, the slides were washed in PBS, incubated with an appropriate biotinylated secondary antibody, washed and incubated with streptavidin-peroxidase (DAKO) according to the manufacturer's protocol. Staining was visualised by adding 3.3'-diaminobenzidine (DAKO) with subsequent counterstaining using haematoxylin. Sections were dehydrated in graded ethanol and embedded in vitro-clud. Samples were only included in the analysis if two or more evaluable cores and were available after sectioning and the immunohistochemical staining procedure. Depending on the respective antibody 14-16 cases with less than two evaluable cores were excluded from the analysis.
Staining intensities were scored semiquantitatively (grade $0=$ no staining, $1=$ weak staining, $2=$ strong staining) by two independent observers with experience in histomorphology and immunohistochemical investigations (H-U V, P A) without knowledge of the clinical data. In case of discrepancies, slides were re-evaluated together and the score was jointly determined. The cut-off for positive tumour cells per core was defined with $>10 \%$ of cells per TMA core. However, in the majority of cases either no tumour cells or almost all tumour cells showed a specific reaction. Since each TMA contained positive and negative samples on one slide; no additional negative controls were used. Established positive controls were used as indicated in Table 2. Figure 1 shows representative examples for staining. For GLUT1, membranous or cytoplasmatic staining is described (Airley et al. 2001). However, its biological function is most likely related to localisation in all membrane. Therefore, only cases with membranous staining (with or without cytoplasmatic staining) were included in the analysis.
Specific staining
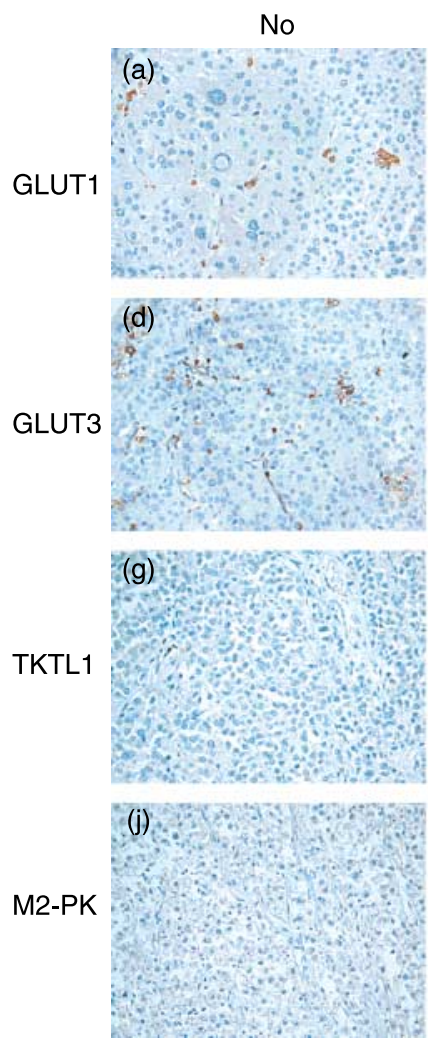

Low
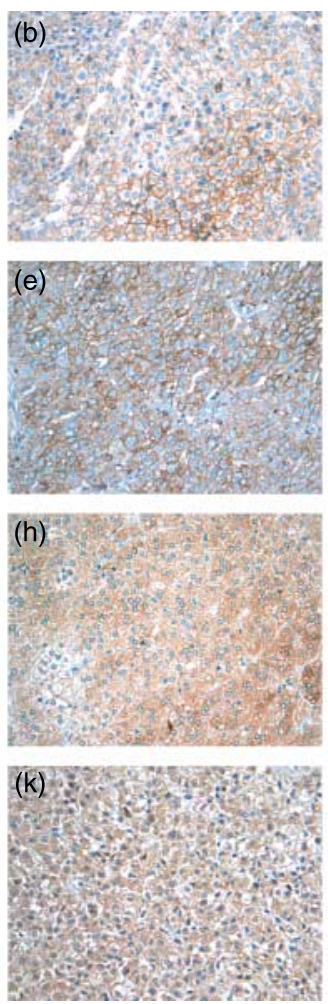

High
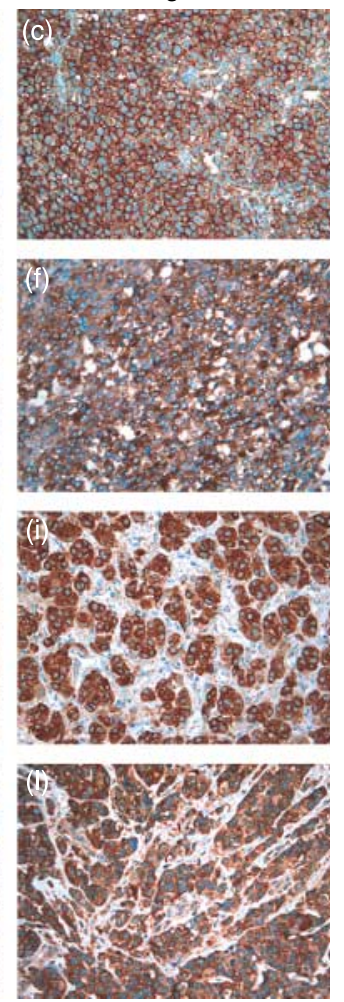

Figure 1 Expression of GLUT1, GLUT3, TKTL1 and M2-PK by immunohistochemistry in representative ACC tissue samples. First, row shows GLUT1-stained sections, second row GLUT3-, third row the TKTL1-, and fourth row the M2-PK-stained sections respectively. In the first column samples without specific staining are shown and in column two and three with low and high staining intensity. Magnification: $\times 100$. 
Table 3 Markers of glucose metabolism in adrenal tissue

\begin{tabular}{|c|c|c|c|}
\hline Staining intensity & No & Low & High \\
\hline \multicolumn{4}{|l|}{ GLUT1 } \\
\hline $\operatorname{ACC}(n=152)$ & 101 & 35 & 16 \\
\hline Adenoma $(n=15)$ & 15 & - & - \\
\hline Normal adrenal gland $(n=4)$ & 4 & - & - \\
\hline \multicolumn{4}{|l|}{ GLUT3 } \\
\hline $\operatorname{ACC}(n=153)$ & 132 & 15 & 6 \\
\hline Adenoma $(n=15)$ & 15 & - & - \\
\hline Normal adrenal gland $(n=4)$ & 4 & - & - \\
\hline \multicolumn{4}{|l|}{ M2-PK } \\
\hline $\operatorname{ACC}(n=151)$ & 27 & 99 & 25 \\
\hline Adenoma $(n=15)$ & - & 11 & 4 \\
\hline Normal adrenal gland $(n=4)$ & - & 3 & 1 \\
\hline \multicolumn{4}{|l|}{ TKTL1 } \\
\hline $\operatorname{ACC}(n=152)$ & 11 & 124 & 17 \\
\hline Adenoma $(n=15)$ & - & 11 & 4 \\
\hline Normal adrenal gland $(n=4)$ & - & 3 & 1 \\
\hline
\end{tabular}

\section{Statistical analysis and clinical correlation}

Characteristics of tumours and patients are presented as means with their respective S.D. values for normally distributed variables. Categorical variables were compared by Fisher's exact test and $\chi^{2}$-test. Survival analysis for ACC patients was calculated using the Kaplan-Meier method and the differences between groups were assessed with log-rank statistics. Overall survival was defined as time elapsed from primary diagnosis of ACC to death or last follow-up visit. Disease-free survival (DFS) was analysed only in patients after radical resection and was defined as time from the date of tumour resection to the first evidence of relapse or last follow-up without evidence for disease. To assess response to cytotoxic drugs, we analysed response to platinum-based chemotherapy in 32 patients. Platinum-based compounds were chosen because they are the most commonly used cytotoxic drugs for ACC (Ahlman et al. 2001, Allolio et al. 2004) and were also the most frequently used drugs in our series. Response was judged according RECIST criteria (Therasse et al. 2000). The Cox proportional hazards model was used for multivariate analysis to test independence from sex, age and tumour stage on survival. The significance level was set at $\alpha=5 \%$ for all comparisons. All statistical tests were done using the SPSS software package (version 15.0.0, Chicago, IL, USA).

\section{Results}

\section{Expression of glucose metabolism markers in adrenal tissues}

The cellular staining pattern of GLUT1, GLUT3, M2PK and TKTL1 in adrenocortical tumours was similar to previous reports for other tumour entities (Fig. 1). GLUT1 and GLUT3 showed a characteristic membranous staining, whereas M2-PK and TKTL1 stained cytoplasmatically. Staining intensity was identical within the different cores of the same tumour sample suggesting homogenous expression of the antigens in the vital tumour regions.

Specific GLUT1 staining was present in 51 out of 152 evaluable ACC samples (33.5\%) including 16 samples $(10.6 \%)$ with strong staining. In none of the adrenal adenomas or normal adrenal glands GLUT1 expression was observed. Similarly, expression of GLUT3 was not present in adenomas or normal adrenal glands, but in 21 out of 153 ACC samples (13.7\%) indicated that the expression of GLUT1 and -3 is associated with a malignant phenotype. By contrast, M2-PK and TKTL1 staining was detectable in all adenomas and normal adrenal glands and in the majority of ACC samples (82.1 and $92.7 \%$ respectively). For details see Table 3.

There was no significant correlation between type of hormone secretion in benign or malignant adrenocortical tumours and GLUT1, GLUT3, M2-PK or TKTL1 expression.

In 19 patients, we had the opportunity to analyse tumour samples from consecutive surgeries (surgery at the time of primary diagnosis and surgery for recurrent or metastatic disease). In the majority of these cases the intensity of GLUT1, GLUT3, M2-PK and TKTL1

Table 4 Expression of GLUT1, GLUT3, M2-PK and TKTL1 and hazard ratio (HR) for death in patients with adrenocortical carcinoma (ACC) after primary surgery

\begin{tabular}{llll}
\hline & $\begin{array}{l}\text { Number of } \\
\text { positive samples }\end{array}$ & Univariate analysis & Multivariate analysis $^{\text {a }}$ \\
\hline GLUT1 $(n=118)$ & $40(34 \%)$ & HR $2.1(1.26-3.14) ; P<0.01$ & HR 1.81 $(1.09-2.98) ; P<0.01$ \\
GLUT3 $(n=118)$ & $14(12 \%)$ & HR $0.56(0.23-1.14) ; P=0.22$ & HR $0.55(0.22-1.38) ; P=0.203$ \\
M2-PK $(n=118)$ & $100(85 \%)$ & HR $0.85(0.44-1.62) ; P=0.622$ & HR $1.01(0.52-1.92) ; P=0.99$ \\
TKTL1 $(n=118)$ & $110(93 \%)$ & HR $1.09(0.39-3.02) ; P=0.86$ & HR $0.67(0.23-1.01) ; P=0.45$ \\
\hline
\end{tabular}

aThe model for the multivariate analysis included age (as a continuous variable), and tumour stage (in three strata; due to the low number of patients in stage 1, these were combined with stage 2 patients to one group) as covariates. 
staining was unchanged. However, in 5, 3, 6 and 2 cases respectively, it was reduced in the specimen of the second surgery and in 4, 0, 1 and 3 cases it was increased.

\section{Glucose metabolism markers and clinical outcome}

For survival analysis, only patients with ACC were included, in whom tumour samples from primary surgery and sufficient clinical data (including followup) were available $(n=130)$. GLUT1 expression was present in 40 out of 118 evaluable samples (33.8\%), GLUT3 in 15 out of $119(12.6 \%)$, M2-PK in 99 out of $117(84.6 \%)$ and TKTL1 in 110 out of 118 samples (93.2\%) (Table 4). In univariate analysis, patients with GLUT1 expression showed increased mortality in comparison with patients without GLUT1 expression (hazard ratio (HR) for death 2.1 (95\% confidence interval (CI) 1.26-3.14). In a multivariate model adjusted for tumour stage and age, GLUT1 expression remained a significant prognostic factor for death (HR 1.81 (1.12-3.07) Table 4), confirming GLUT1 expression as an independent prognostic factor for poor survival in ACC. Similar results were obtained when the analysis was performed using the new tumour staging system proposed by the European adrenal network (ENSAT), which has superior prognostic potential (Fassnacht et al. 2009). There was no association between GLUT1 staining status and tumour stage $(P=0.13)$. In contrast to GLUT1, expression of GLUT3, M2-PK and TKTL1 did not correlate significantly with clinical outcome in ACC (Table 4).

When the patients were stratified according to the staining intensity of GLUT1, there was a strong association between the grade of GLUT1 expression and survival with and without adjusting for tumour stage and age $(P<0.01 ;$ Fig. $2 \mathrm{a})$. In univariate analysis, patients with weak GLUT1 staining had a HR for death of $1.45(0.81-2.96 ; P=0.22)$, whereas patients with strong GLUT1 staining had a HR of 6.34 (3.10-12.90; $P<0.01)$. Similarly, GLUT1 expression was associated with a shorter DFS after radical surgical resection $(n=76)$; the median DFS in patients without GLUT1 staining was 41.9 months compared with only 22.2 months in patients with GLUT1 expression (HR 2.2, $1.12-4.42 ; P=0.01)$. Again, after classifying patients according to their staining intensity, there was an association between the intensity of GLUT1 expression and shorter DFS: in comparison with patients without GLUT1 expression, the HR for tumour recurrence in patient with low GLUT1 staining
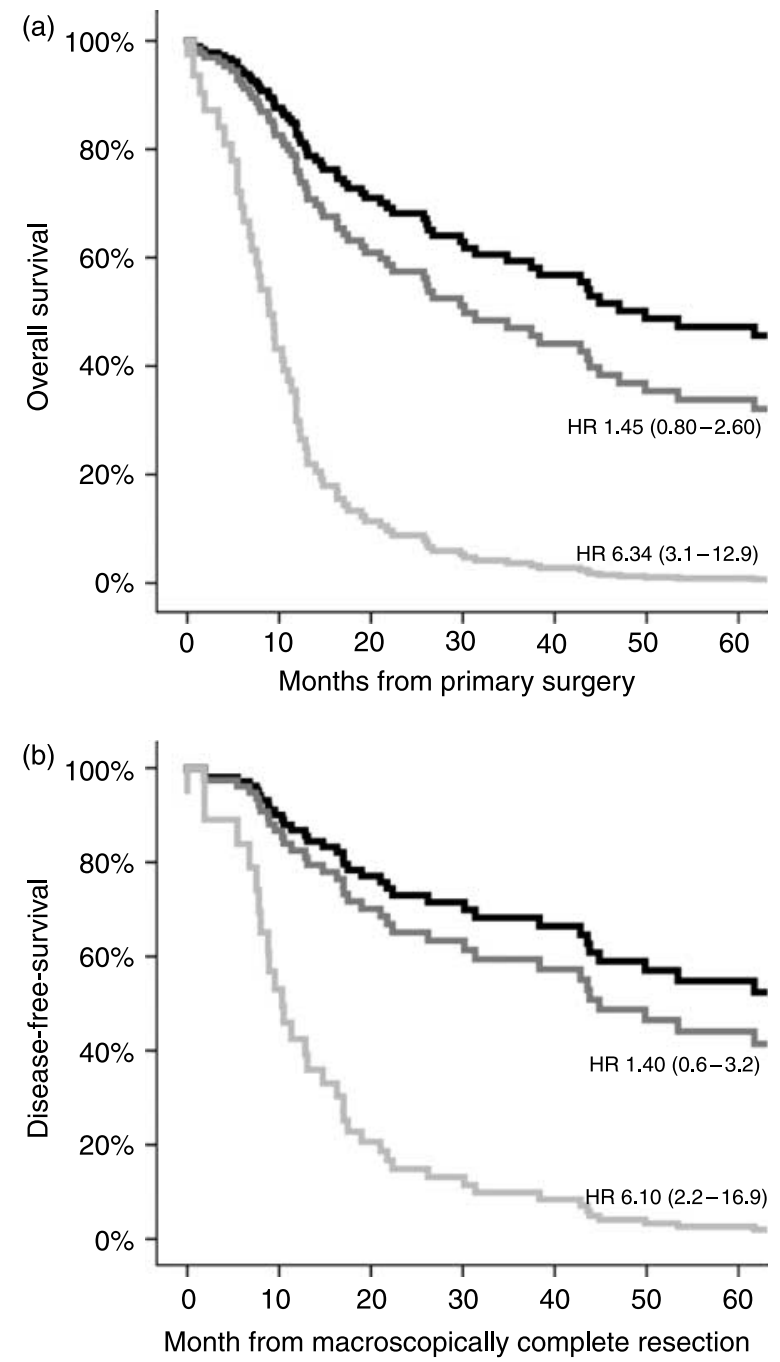

Figure 2 (a) Cox regression survival curves for 118 patients with ACC after primary surgery, substratified in high (light grey), low (dark grey), and no GLUT1 expression (black). (b) Cox regression of disease-free survival curves for 76 patients with ACC after macroscopically complete resection, according to high (black), low (dark grey), and no GLUT1 expression (light grey).

was $1.36(0.59-3.19 ; P=0.47)$, whereas ACC patients with high GLUT1 staining had a HR of 6.01 (2.1616.94; $P=0.01$ ) (Fig. 2b). In patients presented with distant metastases at diagnosis $(n=28)$ GLUT1 was also of a predictive value. Patients with high GLUT1 expression had a considerably shorter overall survival than patients with low or no GLUT1 expression (median survival: 7.97 vs 27.80 vs 42.25 months) with a HR of $5.31(1.80-15.62 ; P<0.01)$ for patients with high GLUT1 expression.

In addition, we analysed 32 patients, who received platinum-based chemotherapy. However, no relation between tumour response and GLUT1 was found. 


\section{Discussion}

The main finding of our study is the strong prognostic potential of membranous GLUT1 protein expression for patient outcome in ACC. This predictive quality concerns both patients with early tumour stages and patients with advanced disease. Although both, cytoplasmatic and membranous GLUT staining was observed in different tumour samples, we have evaluated only tumours with membranous staining because the biological function of GLUT as a glucose transporter (Kato et al. 2002).

As the tumour stage is currently the only accepted prognostic marker, our observation is of significant clinical relevance as the tumour stage. Recent studies that have tried to establish prognostic markers for ACC using histomorphology or immunohistochemistry suffered from small sample size or did not adjust their findings for tumour stage (Evans \& Vassilopoulou-Sellin 1996, Stojadinovic et al. 2002, Assie et al. 2007). The largest published study included 67 ACC samples from a single centre in the US. Low mitotic index was the only marker with some prognostic value in multivariate analysis (Stojadinovic et al. 2002). Similar results were found by Assie et al. (2007) analysing 44 selected patients with metastasised ACC. For this reason, we have also analysed the mitotic index in the 130 ACC samples used for survival analysis, and compared its prognostic value with GLUT1. However, in our cohort the mitotic index did not correlate significantly with overall survival (univariate analysis HR 1.24, 95\% (CI 0.95-1.62) and multivariate analysis, adjusted for age and tumour stage HR 1.22, 95\% (CI 0.92-1.59)). Accordingly, the impact of high GLUT1 expression on prognosis in our series is even more remarkable. None of the 12 evaluable patients with high GLUT1 (including one patient in stage I and four in stage II) are still alive and only one survived for 21 months, whereas all others died within the first year after diagnosis. By contrast, $51 \%$ of patients without GLUT1 expression are still alive with a median survival of 62 months. Therefore, patients with high GLUT1 expression seem to be at very high risk for ACC-related death and it might be justified to treat them aggressively independent of their tumour stage at diagnosis. In contrast to a publication in ovarian cancer (Cantuaria et al. 2001), we could not find any correlation of GLUT1 expression and response to cytotoxic chemotherapy. However, this analysis has several limitations: mainly the small sample size with only 32 patients and the fact that we have investigated only platinum-based regimens.
Although the number of non-malignant adrenal tissue samples in our series were small $(n=19)$, it is important to note that in none of these samples GLUT1 or GLUT3 protein was detected. Thus, the expression of GLUT1 or GLUT3 in ACC seems to be highly specific for the malignant phenotype. By contrast, sensitivity for diagnosing malignancy is rather limited, as only 33.5 and $13.7 \%$ of ACC cases examined in the current study were positive for GLUT1 and GLUT3 respectively. In 11 out of 101 GLUT1 negative samples, GLUT3 expression was found, hence, about $60 \%$ of ACC samples are negative for both transporters. The reasons for this may be various: in these ACCs other GLUTs may be responsible for the supply of glucose or tumours are less dependent on glucose metabolism as their main source of energy. In addition, we can not fully exclude that heterogeneity of GLUT1 expression within tumour samples may have contributed to the low frequency of positive GLUT staining in our TMAs. However, in a subset of specimens we have evaluated also the staining in regular tissue slides of ACCs. The results correlated fully with the corresponding spots on the microarray, making this explanation unlikely. Many ACCs are harbouring necrotic areas that are more likely to lead to GLUT1 expression locally. However, these areas are often difficult to judge reliably by immunohistochemistry. Therefore, we have restricted our analysis to areas without necrosis.

The expression and functionality of GLUTs in tumours are probably related to a number of factors. In this context, the role of oncogenes and tumour hypoxia has generated considerable interest (Dang et al. 1997).

It has been proposed that the activation of oncogenes results in increased transcription of GLUTs and glycolytic enzyme genes (Baron-Delage et al. 1996, Dang et al. 1997, Onetti et al. 1997, Osthus et al. 2000, Chen et al. 2001). Therefore, the activation of different oncogenes may explain the overexpression of different metabolic pathways leading to phenotypic heterogeneity concerning expression of GLUTs.

This may explain why glucose utilisation could be measured by $18 \mathrm{~F}$-fluordesoxyglucose (FDG-PET) also in these carcinomas, not expressing GLUT1 or GLUT3. To analyse the correlation between FDG-maximal standardised uptake values and GLUT expression in ACC, further investigations will be required.

In contrast to the glucose, transporter 1 and 3, TKTL1 and M2-PK were present in all non-malignant adrenal samples and the in vast majority of ACCs suggesting that these factors do not play an important 
role in adrenocortical malignancy. Accordingly, TKTL1 and M2-PK harbour no prognostic potential. The same was true for GLUT3 expression, indicating that hypoxia-induced up-regulation of GLUT3 is of minor importance for prognosis in ACC.

Our results emphasise the molecular heterogeneity of ACC and indicate the need for targeted molecular therapies tailored to the individual patient. Several researchers have proposed the inhibition of GLUTs as a potential therapeutic target (Fung et al. 1986, Grover-McKay et al. 1998, Cantuaria et al. 2001). However, GLUT1 is also abundantly expressed in endothelial cells in normal tissues (North et al. 2000), in brain microvessels (Simpson et al. 1999) and in other blood tissue barriers. Thus, targeting of GLUT1 may be highly toxic to cells dependent on nutrient provision at these sites and the side effects of such an approach will be probably unacceptable. However, for future use of hypoxia-selective therapies in cancer (Patterson et al. 2002), genes or proteins inducible via the HIF pathway like GLUT1 may become highly valuable markers of tumour hypoxia. Additionally, it is known, that some members of the GLUT/SLC2A family exist in different molecular forms resulting from splice variants (Augustin et al. 2004), differential glycosylation and other post-translational modifications. This may create opportunities to develop novel and specific compounds to inhibit specific molecular variants of GLUT1 overexpression in ACC and other cancers. Since only paraffin embedded ACC samples were available for our analysis, we could not test this hypothesis in this study.

In conclusion, our results suggest that GLUT1 immunostaining may provide important prognostic information in patients with ACC, independent of tumour stage. We hypothesise, that high GLUT1 expression in ACC indicates increased glucose uptake and utilisation, which correlates with aggressive behaviour and requires an aggressive therapeutic strategy. Further studies analysing the association of GLUT1 with biologic markers of proliferation, oncogenes and growth factors will allow a more in-depth understanding of the biologic significance of GLUT1 overexpression and may guide targeted therapies.

\section{Declaration of interest}

The authors declare that there is no conflict of interest that could be perceived as prejudicing the impartiality of the research reported.

\section{Funding}

This study was supported by grants of the Deutsche Krebshilfe (\# 106080 to B A and M F and grant \# 107111 to $\mathrm{M} \mathrm{F}$ ) and the German Ministry of Research BMBF (\#01KG0501 to B A and M F).

\section{Acknowledgements}

This study was part of the German adrenal network GANIMED (German Adrenal Network Improving Treatment and Medical Education). We are grateful to all colleagues who provided tumour material and clinical data for the German ACC registry: the following pathologists provided tumour material from two or more patients for the tissue array: Wolfgang Saeger (Marienkrankenhaus Hamburg); Gerhard Seitz (Klinikum Bamberg); Harald Stein, Manfred Dietel, (Charite University Berlin); Gerhard Mall (Klinikum Darmstadt); Helmut Erich Gabbert (University Hospital Duesseldorf); Werner Schmid (University of Essen); Steffen Hauptmann (Martin-Luther University of Halle); Peter Schirrmacher (University of Heidelberg); Alfred C Feller (University of Luebeck); C James Kirkpatrick (University of Mainz); Roland Moll (University of Marburg); Cyrus Tschahargane (Lukaskrankenhaus, Neuss); Rainer Horst Krech (Klinikum Osnabrueck); Ferdinand Hofstaedter (University of Regensburg); Andrea M Gassel (Leopoldina Hospital Schweinfurt). The following hospitals/clinicians contributed clinical data from three or more investigated patients: Marcus Quinkler; Wolfgang Oelkers (University Hospital Charite Berlin); Holger Willenberg (University of Düsseldorf); Peter Langer (University Hospital of Marburg); Christian Fottner (University Hospital Mainz); Michael Brauckhoff (University Hospital Halle); Felix Beuschlein (University of Freiburg); Horst L Fehm (University Hospital Luebeck); Dagmar Führer (University Hospital Leipzig); Stefan Petersenn (University Hospital Essen). We are grateful to Wolfgang Saeger (Marienkrankenhaus Hamburg) who confirmed the histopathological diagnoses of all tumour samples. We appreciate the support of Uwe Maeder (Tumour Centre, University Hospital Wuerzburg) in establishing the German ACC registry database and are thankful to Michaela Haaf for documentation.

\section{References}

Ahlman H, Khorram-Manesh A, Jansson S, Wangberg B, Nilsson O, Jacobsson CE \& Lindstedt S 2001 Cytotoxic treatment of adrenocortical carcinoma. World Journal of Surgery 25 927-933.

Airley R, Loncaster J, Davidson S, Bromley M, Roberts S, Patterson A, Hunter R, Stratford I \& West C 2001 Glucose transporter GLUT-1 expression correlates with tumor hypoxia and predicts metastasis-free survival in advanced carcinoma of the cervix. Clinical Cancer Research 7 928-934. 
Allolio B \& Fassnacht M 2006 Clinical review: adrenocortical carcinoma: clinical update. Journal of Clinical Endocrinology and Metabolism 91 2027-2037.

Allolio B, Hahner S, Weismann D \& Fassnacht M 2004 Management of adrenocortical carcinoma. Clinical Endocrinology 60 273-287.

Assie G, Antoni G, Tissier F, Caillou B, Abiven G, Gicquel C, Leboulleux S, Travagli JP, Dromain C, Bertagna X et al. 2007 Prognostic parameters of metastatic adrenocortical carcinoma. Journal of Clinical Endocrinology and Metabolism 92 148-154.

Augustin R, Carayannopoulos MO, Dowd LO, Phay JE, Moley JF \& Moley KH 2004 Identification and characterization of human glucose transporter-like protein-9 (GLUT9): alternative splicing alters trafficking. Journal of Biological Chemistry 279 16229-16236.

Baron-Delage S, Mahraoui L, Cadoret A, Veissiere D, Taillemite JL, Chastre E, Gespach C, Zweibaum A, Capeau J, Brot-Laroche E et al. 1996 Deregulation of hexose transporter expression in Caco-2 cells by ras and polyoma middle T oncogenes. American Journal of Physiology 270 G314-G323.

Barzon L, Fallo F, Sonino N, Daniele O \& Boscaro M 1997 Adrenocortical carcinoma: experience in 45 patients. Oncology 54 490-496.

Cantuaria G, Fagotti A, Ferrandina G, Magalhaes A, Nadji M, Angioli R, Penalver M, Mancuso S \& Scambia G 2001 GLUT-1 expression in ovarian carcinoma: association with survival and response to chemotherapy. Cancer 92 1144-1150.

Chen C, Pore N, Behrooz A, Ismail-Beigi F \& Maity A 2001 Regulation of glut1 mRNA by hypoxia-inducible factor1. Interaction between H-ras and hypoxia. Journal of Biological Chemistry 276 9519-9525.

Comin-Anduix B, Boren J, Martinez S, Moro C, Centelles JJ, Trebukhina R, Petushok N, Lee WN, Boros LG \& Cascante M 2001 The effect of thiamine supplementation on tumour proliferation. A metabolic control analysis study. European Journal of Biochemistry 268 4177-4182.

Coy JF, Dressler D, Wilde J \& Schubert P 2005 Mutations in the transketolase-like gene TKTL1: clinical implications for neurodegenerative diseases, diabetes and cancer. Clinical Laboratory 51 257-273.

Dackiw AP, Lee JE, Gagel RF \& Evans DB 2001 Adrenal cortical carcinoma. World Journal of Surgery 25 914-926.

Dang CV, Lewis BC, Dolde C, Dang G \& Shim H 1997 Oncogenes in tumor metabolism, tumorigenesis, and apoptosis. Journal of Bioenergetics and Biomembranes 29 345-354.

DeLellis RA, Lloyd RV, Heitz PU \& Eng C 2004 Tumour of the pituitary gland. In World Health Organization Classification of Tumours. Pathology and Genetics of Tumours of Endocrine Organs, pp 10-47. Lyon, France: IARC Press.

Eckert AW, Lautner MH, Taubert H, Schubert J \& Bilkenroth U 2008 Expression of GLUT-1 is a prognostic marker for oral squamous cell carcinoma patients. Oncology Reports 20 1381-1385.
Evans HL \& Vassilopoulou-Sellin R 1996 Adrenal cortical neoplasms. A study of 56 cases. American Journal of Clinical Pathology 105 76-86.

Fassnacht M, Kenn W \& Allolio B 2004 Adrenal tumors: how to establish malignancy? Journal of Endocrinological Investigation 27 387-399.

Fassnacht M, Hahner S, Polat B, Koschker AC, Kenn W, Flentje M \& Allolio B 2006 Efficacy of adjuvant radiotherapy of the tumor bed on local recurrence of adrenocortical carcinoma. Journal of Clinical Endocrinology and Metabolism 91 4501-4504.

Fassnacht M, Johanssen S, Quinkler M, Bucsky P, Willenberg HS, Beuschlein F, Terzolo M, Mueller HH, Hahner S \& Allolio B 2009 Limited prognostic value of the 2004 International Union Against Cancer staging classification for adrenocortical carcinoma: proposal for a Revised TNM Classification. Cancer 115 243-250.

Fung KP, Ng SW, Ha DK \& Choy YM 1986 Suppression of glucose transport of Ehrlich ascites tumour cell by interferon inducers. Chemotherapy 32 458-463.

Grover-McKay M, Walsh SA, Seftor EA, Thomas PA \& Hendrix MJ 1998 Role for glucose transporter 1 protein in human breast cancer. Pathology Oncology Research 4 115-120.

Kato H, Takita J, Miyazaki T, Nakajima M, Fukai Y, Masuda N, Fukuchi M, Manda R, Ojima H, Tsukada K et al. 2002 GLUT-1 glucose transporter expression in esophageal squamous cell carcinoma is associated with tumor aggressiveness. Anticancer Research 22 2635-2639.

Koschker AC, Fassnacht M, Hahner S, Weismann D \& Allolio B 2006 Adrenocortical carcinoma - improving patient care by establishing new structures. Experimental and Clinical Endocrinology \& Diabetes 114 45-51.

Langbein S, Zerilli M, Zur Hausen A, Staiger W, Rensch-Boschert K, Lukan N, Popa J, Ternullo MP, Steidler A, Weiss C et al. 2006 Expression of transketolase TKTL1 predicts colon and urothelial cancer patient survival: Warburg effect reinterpreted. British Journal of Cancer 94 578-585.

Libe R, Fratticci A \& Bertherat J 2007 Adrenocortical cancer: pathophysiology and clinical management. Endocrine-Related Cancer 14 13-28.

Mazurek S, Boschek CB, Hugo F \& Eigenbrodt E 2005 Pyruvate kinase type M2 and its role in tumor growth and spreading. Seminars in Cancer Biology 15 300-308.

North PE, Waner M, Mizeracki A \& Mihm MC Jr 2000 GLUT1: a newly discovered immunohistochemical marker for juvenile hemangiomas. Human Pathology 31 11-22.

Onetti R, Baulida J \& Bassols A 1997 Increased glucose transport in ras-transformed fibroblasts: a possible role for $\mathrm{N}$-glycosylation of GLUT1. FEBS Letters $\mathbf{4 0 7}$ 267-270.

Osthus RC, Shim H, Kim S, Li Q, Reddy R, Mukherjee M, Xu Y, Wonsey D, Lee LA \& Dang CV 2000 
Deregulation of glucose transporter 1 and glycolytic gene expression by c-Myc. Journal of Biological Chemistry 275 21797-21800.

Patterson AV, Williams KJ, Cowen RL, Jaffar M, Telfer BA, Saunders M, Airley R, Honess D, van der Kogel AJ, Wolf CR et al. 2002 Oxygen-sensitive enzyme-prodrug gene therapy for the eradication of radiation-resistant solid tumours. Gene Therapy 9 946-954.

Rais B, Comin B, Puigjaner J, Brandes JL, Creppy E, Saboureau D, Ennamany R, Lee WN, Boros LG \& Cascante M 1999 Oxythiamine and dehydroepiandrosterone induce a G1 phase cycle arrest in Ehrlich's tumor cells through inhibition of the pentose cycle. FEBS Letters 456 113-118.

Reske SN, Grillenberger KG, Glatting G, Port M, Hildebrandt M, Gansauge F \& Beger HG 1997 Overexpression of glucose transporter 1 and increased FDG uptake in pancreatic carcinoma. Journal of Nuclear Medicine 38 1344-1348.

Schteingart DE, Doherty GM, Gauger PG, Giordano TJ, Hammer GD, Korobkin M \& Worden FP 2005 Management of patients with adrenal cancer: recommendations of an international consensus conference. EndocrineRelated Cancer 12 667-680.

Simpson IA, Appel NM, Hokari M, Oki J, Holman GD, Maher F, Koehler-Stec EM, Vannucci SJ \& Smith QR 1999 Blood-brain barrier glucose transporter: effects of hypo- and hyperglycemia revisited. Journal of Neurochemistry 72 238-247.

Smith TA 1999 Facilitative glucose transporter expression in human cancer tissue. British Journal of Biomedical Science 56 285-292.

Stojadinovic A, Ghossein RA, Hoos A, Nissan A, Marshall D, Dudas M, Cordon-Cardo C, Jaques DP \& Brennan MF 2002 Adrenocortical carcinoma: clinical, morphologic, and molecular characterization. Journal of Clinical Oncology 20 941-950.
Therasse P, Arbuck SG, Eisenhauer EA, Wanders J, Kaplan RS, Rubinstein L, Verweij J, Van Glabbeke M, van Oosterom AT, Christian MC et al. $2000 \mathrm{New}$ guidelines to evaluate the response to treatment in solid tumors. European Organization for Research and Treatment of Cancer, National Cancer Institute of the United States, National Cancer Institute of Canada. Journal of the National Cancer Institute 92 205-216.

Urano N, Fujiwara Y, Doki Y, Tsujie M, Yamamoto H, Miyata H, Takiguchi S, Yasuda T, Yano M \& Monden M 2006 Overexpression of hypoxia-inducible factor-1 alpha in gastric adenocarcinoma. Gastric Cancer 9 44-49.

Vassilopoulou-Sellin R \& Schultz PN 2001 Adrenocortical carcinoma. Clinical outcome at the end of the 20th century. Cancer 92 1113-1121.

Warburg O 1956 On the origin of cancer cells. Science $\mathbf{1 2 3}$ 309-314.

Weiss LM, Medeiros LJ \& Vickery AL Jr 1989 Pathologic features of prognostic significance in adrenocortical carcinoma. American Journal of Surgical Pathology 13 202-206.

Wincewicz A, Sulkowska M, Koda M \& Sulkowski S 2007 Clinicopathological significance and linkage of the distribution of HIF-1alpha and GLUT-1 in human primary colorectal cancer. Pathology Oncology Research 13 15-20.

Yoo BC, Ku JL, Hong SH, Shin YK, Park SY, Kim HK \& Park JG 2004 Decreased pyruvate kinase M2 activity linked to cisplatin resistance in human gastric carcinoma cell lines. International Journal of Cancer 108 532-539.

Zhou S, Wang S, Wu Q, Fan J \& Wang Q 2008 Expression of glucose transporter- 1 and -3 in the head and neck carcinoma - the correlation of the expression with the biological behaviors. ORL; Journal for Oto-rhino-laryngology and its Related Specialties $\mathbf{7 0}$ 189-194. 